\title{
Photobiomodulation Therapy in Hearing Related Aspects: an Integrative Bibliometric Study
}

Amanda Rodrigues Scheffer ${ }^{1 *}$ Maria Fernanda Capoani Garcia Mondelli² Renata da Silva Cardoso Rocha Tavares ${ }^{3}$

\begin{abstract}
Introduction: In the last decades have been increasing cases of inner ear disorders and among them is included hearing loss, tinnitus, and vertigo, on that way the demand for new treatments is growing, with low-level laser therapy (LLLT) being a highly targeted treatment due to its non-invasive nature.

Objectives: The present study aims to investigate the studies of low level laser (LLL) and audiology in recent years.

Materials and Methods: The quantitative bibliometric study was performed by searching scientific articles in the Web of Science Clarivate Analytics database. The association of the words "low level laser therapy" and "hearing" was used to construct the sample.

Results: The most recurrent objectives analyzed the action of LLLT regarding its effectiveness, with predominantly positive results in the effectiveness of the treatment of hearing problems such as hearing loss, tinnitus and vertigo.

Conclusions: LLL is a great tool for professionals who take care of human hearing, helping in the recovery of patients with hearing problems in a noninvasive or drug way, thus improving the functions in which the TBLI is applied
\end{abstract}

Keywords: Tinnitus; Audiology; Low level laser therapy; Hearing Disorders; Bibliometrics.

${ }^{1}$ Department of audiology and speech Therapy University of São Paulo (USP), Brazil

${ }^{2}$ Department of Audiology and Speech Therapy, counselor registered at the Postgraduate Program in Audiology and Speech, University of São Paulo (USP), Brazil

${ }^{3}$ Department of audiologist, University of São Paulo (USP), Brazil 


\section{INTRODUCTION}

Hearing impairment is a silent and invisible problem that often goes unnoticed by the individual and the family itself, which is considered a major public health problem since it causes numerous losses to the development of human beings ${ }^{1}$. Over the years, there has been an increase in the demand for audiologist professionals, since the geriatric population has been growing rapidly and along with it, hearing and balance problems, in addition to speech, language and swallowing disorders also increase. In the last decades has been increasing cases of inner ear disorders and among them is included hearing loss, tinnitus, and vertigo and the demands of that kind of patients are predominantly older, thus, the audiologist has the role of minimizing the deficiency and maximizing the patient's daily function ${ }^{2-4}$. Thus, the demand for new treatments is growing, with Low Level Laser Therapy (LLLT) being a highly targeted treatment due to its non-invasive nature ${ }^{5}$. The acronym LASER has its origin in the English language, abbreviating "light amplification by stimulated emission of radiation" and is defined as a monochromatic, intense, coherent and collimated light source, whose radiation emission is done by stimulating the external field, with varied applications and are classified as high (applied to the removal, cutting and coagulation of tissues) and low (applied to tissue repair processes, such as muscle, joint, nerve, bone and skin injuries) ${ }^{6}$. Its anti-inflammatory action is achieved through microcirculation acceleration, which determines the types of changes in the hydrostatic pressure of the capillaries, with absorption of edema and inactivation of the intermediate catabolics ${ }^{7}$. Low-level laser therapy (LLLT), sometimes known as low-intensity light therapy or photobiomodulation Therapy (PBMT), is a light therapy with a photochemical, non-thermal effect, since light triggers biochemical changes in cells, similar to the process of photosynthesis in plants, where photons are absorbed by cellular photoreceptors and triggers chemical changes ${ }^{8}$. Researchers believe that mitochondria play an important role in the generation and metabolism of energy and are involved in current research on the mechanism of the effects of photobiomodulation therapy. It is proposed that cytochrome c oxidase (Cco) is the primary photoacceptor for the red and infrared light band in mammalian cells, as these longer wavelengths have more effective tissue penetration compared to the light bands blue or green, being absorbed by hemoglobin more efficiently ${ }^{9,10}$. Because it is an efficient, non-invasive, low-cost and safe tool, capable of promoting anti-inflammatory effects, epithelial and fibroblast proliferation, healing, collagen synthesis and deposition, revascularization, wound contraction, reducing pain, among others, the low intensity laser that is already used in the routine of medical, dental and physiotherapeutic treatments, has become the target of areas of interest in the sciences of speech, language and hearing ${ }^{11,12}$. The therapeutic effects of the Low Level Laser (LLL) have already been presented in the audiology area as in sensorineural hearing loss since it has several etiologies in human patients ${ }^{13}$. Experiments carried out by researchers at the University of Dankook, in South Korea, showed that LLLT can not only prevent cochlear damage caused by exposure to noise, but can also become a treatment for noise-induced hearing loss ${ }^{14}$. Other researchers also agree with these results, showing that PBMT represents an effective tool to control and limit cochlear oxidative stress and the induction of cochlear inflammation located in the organ of Corti, making it a therapeutic alternative to deal with high levels oxidative stress in hearing cells exposed to ototoxic drugs or resulting from exposure to occupational noise ${ }^{15}$. In another study, the application of transmeatal photobiomodulation allowed the functional recovery of the vestibular system of rats with bilateral ototoxic vestibulopathy, showing that the treatment, in addition to being non-invasive and with minimal complications, can be promising for vestibular changes ${ }^{16}$. When applied to tinnitus, it has been showing controversial results. While some researchers conclude that after low-level laser treatment, individuals did not show improvement in tinnitus ${ }^{17-19}$ (17), (18), (19), others show that tinnitus can improve and even disappear after laser treatment $\mathrm{t}^{20-24}$. Since the audiologist is part of the team interested in hearing problems, the interest in joining the group of professionals who use this therapeutic resource has increased over the years ${ }^{25}$. Therefore, the intention of this article is to search the literature for the findings through the bibliometric analysis of the articles in the Web of Science - Clarivate Analytics database using the association of the words "low Level laser therapy" and "hearing", being selected the articles that have in the title the descriptor searched or correlated to some area of audiology.

\section{MATERIALS AND METHODS}

The referring quantitative study of bibliometric character was carried out by searching for scientific articles in the database of Web of Science - Clarivate Analytics. The association of the words "low level laser therapy" and "hearing" was used to construct the sample. As inclusion criteria, we chose to limit the sample to only articles, using the filters: "Document type - article" and English language. As it is a review article, there was no need to submit the study to the Research Ethics Committee. Eight articles from the sample were excluded because they did not fit the theme, did not have the descriptors used, or were not relevant to the research. The total number of articles analyzed in the research was 14 and after selecting the most relevant articles, the sample was considered to be of excellent scientific level. According to Souza and Kerbauy ${ }^{26}$ the quantitative approach is based on generalizing and objectifying the results, distancing the subject from the object, as well as making the researcher neutral in order to ensure and legitimize the scientific research data. The bibliometric study is composed of techniques that aim to quantify the written communication process. Such studies have become popular and their adherence in the health area is a national and international trend, since a large amount of bibliographic material that 
is produced and made available today, which facilitates the understanding of researchers, and may even show future paths of research ${ }^{27,28}$ this study, data were recorded in Microsoft Office Excel, tabulated and described according to the descriptive statistical analysis of the most significant items in relation to the articles read, such as: authors, descriptors, year of publication, journals, objectives, methods, results, conclusions, limitations, suggestions for future studies.

\section{RESULTS AND DISCUSSION}

Fourteen articles were selected that fit the proposed theme and were relevant to the purpose of this research. Regarding the journals that published the articles, the one that had the largest publication of articles was Lasers in Medical Science ( $\mathrm{n}=4,28.6 \%$ ), followed by the Journal of Biomedical Optics ( $n=2,14.3 \%)$ and Journal of Biophotonics ( $n=2,14.3 \%)$. The other publications ( $n$ $=6,42.6 \%$ ) are distributed among 6 different journals, with an impact factor ranging from 1,219 to 3,768 . (Figure 1) In relation to the publication timeline, articles were published between 2001 and 2019, with the years 2012, 2013, 2015 and 2019 adding the largest number of publications about this topic $(57,2 \%)$. Although the article with the longest publication time in the sample is from 2001, it is known that Low Level Laser Therapy (LLLT) has been used for 20 years in Europe for the treatment of pain and tissue repair, and the interest this treatment has grown over the years ${ }^{29,30}$. Regarding the authors who most published on the topic can be seen, highlighting researchers Phil-Sang Chung $(n=6)$ and Jae Yun Jung $(n=6)$, followed by Min Young Lee $(n=4)$. As for the descriptors, 36 were identified, with the terms Low Level Laser Therapy, Photobiomodulation and Noise Induced Hearing Loss the ones that appeared most frequently, as shown in (Figure 2). It is important to highlight that the first two most used descriptors: Low Level Laser Therapy, Photobiomodulation may be present in varied contexts and not only related to its application in audiology, being to limit the search using descriptors together to direct the results (Figure 2). Regarding the study methods, it is possible to observe one quali-quantitative article ( $\mathrm{n}=$
$1,7,1 \%)$ while the use of quantitative methodology of an experimental nature ( $n=13,92.9 \%)$ stands out, since when it comes to an experiment, measures and protocols are necessary, as well as the generalization of data to arrive at hypotheses and results ${ }^{31}$. About to objectives of the articles, they were divided into two categories, researching results with the use of LLL ( $n=13,92,7 \%$ ) and assessing dosimetry $(n=1,7,1 \%)$, in which there was a tendency to research the results of treatments with the use of LLL. It is observed that the interest in conducting research with photobiomodulation (PBM) as a nonmedication alternative for hearing problems is a trend. With the increase in cases of diseases of the inner ear in the last decade, in particular hearing loss, tinnitus and vertigo, the use of LLLT has been sought because it is a non-invasive, painless treatment that does not cause discomfort to the patient ${ }^{32}$. In relation to the classification regarding the demand for laser application, there were 10 divisions, and the ones that stood out the most were regarding the application for tinnitus $(n=4)$, hearing loss $(n=3)$ and exposure to noise $(n=2)$. It is explained that some fit more than one application demand (Figure 3). It is known that hearing can degenerate with age, or can be compromised at birth or when young, due to exposure to noise, ototoxic drugs or genetic problems, thus basic and clinical studies on the use of PBM to protect against hearing loss, tinnitus and vestibular dysfunction in animals and patients have been published, with the intention of finding out whether the treatment is effective or not ${ }^{33}$. About the application protocols used in the research methods of the articles, there was a difference in the application site, the type of laser, the wavelength and the sample configuration. About the application site, transtympanic irradiation ( $n=9,64.3 \%$ ) was the most recurrent, followed by irradiation directly in auditory rodent cells ( $n=3,21,4 \%$ ), transtympanic + mastoid $(n=1,7,1 \%)$ and finally just on the mastoid $(n=1,7,1 \%)$. It is known that irradiation via the auditory canal penetrates more easily and reaches the cochlea more effectively in relation to the mastoid bone, according to the Schuller's X-ray technique ${ }^{34}$. In addition, in vitro research stands out, since there are limitations to this therapy due to the

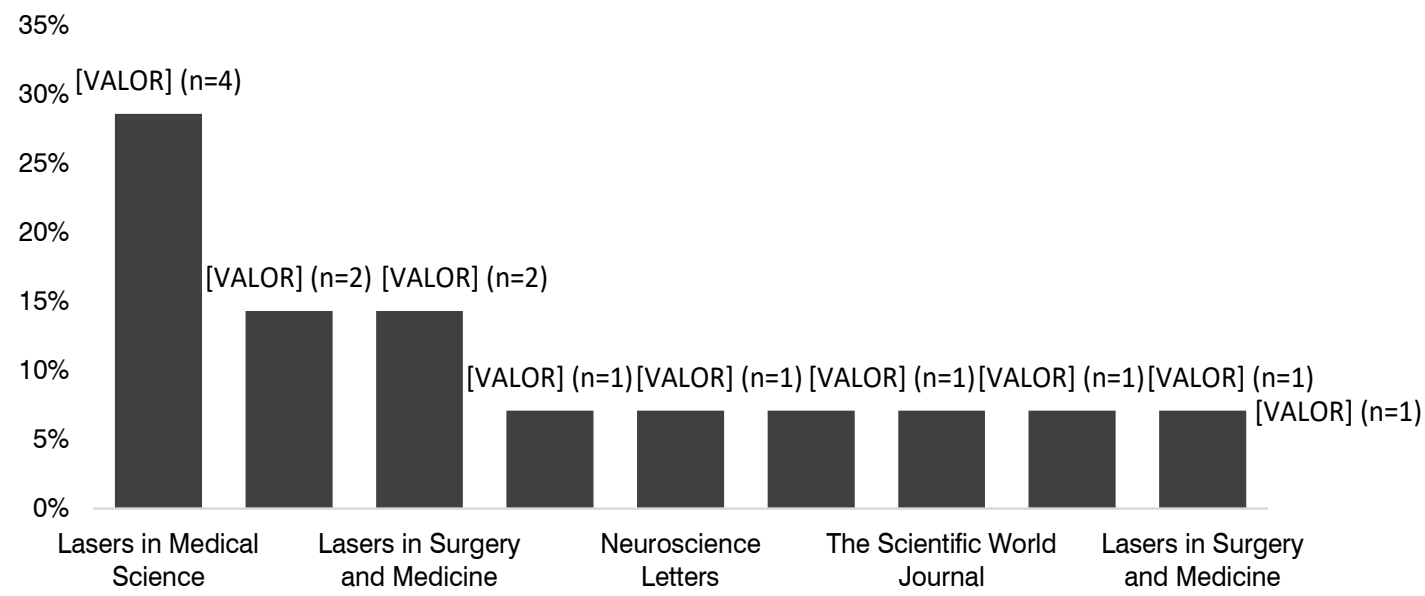

Figure 1: Journals that published on the topic $(n=14)$. 


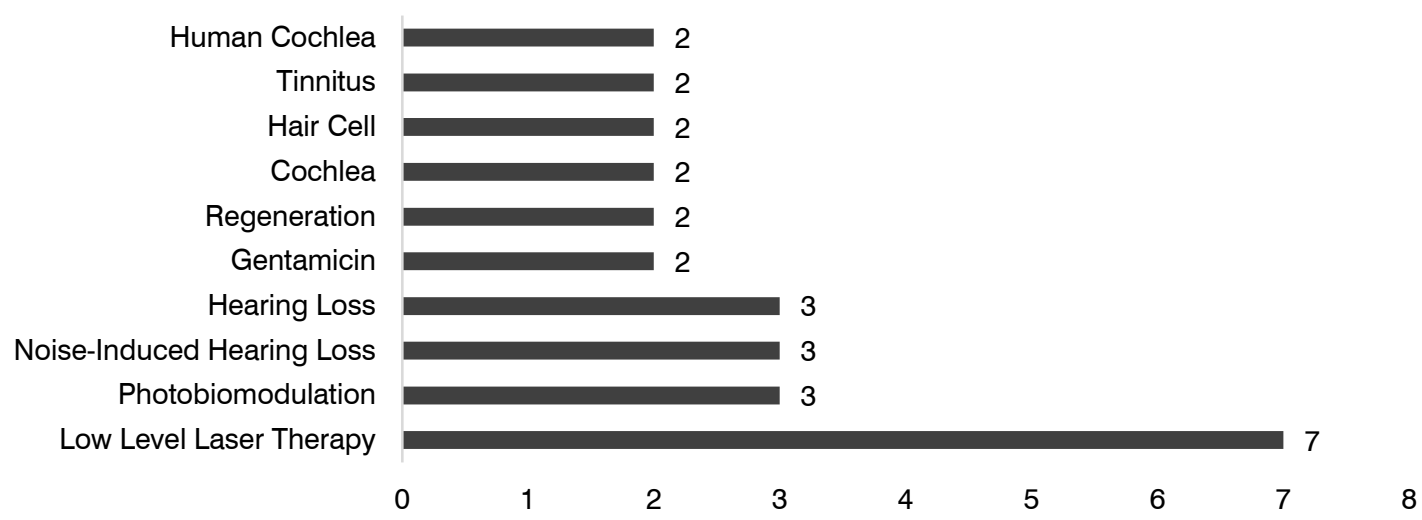

Figure 2: Most used descriptors (Note: This chart shows the ten terms that were most recurring).

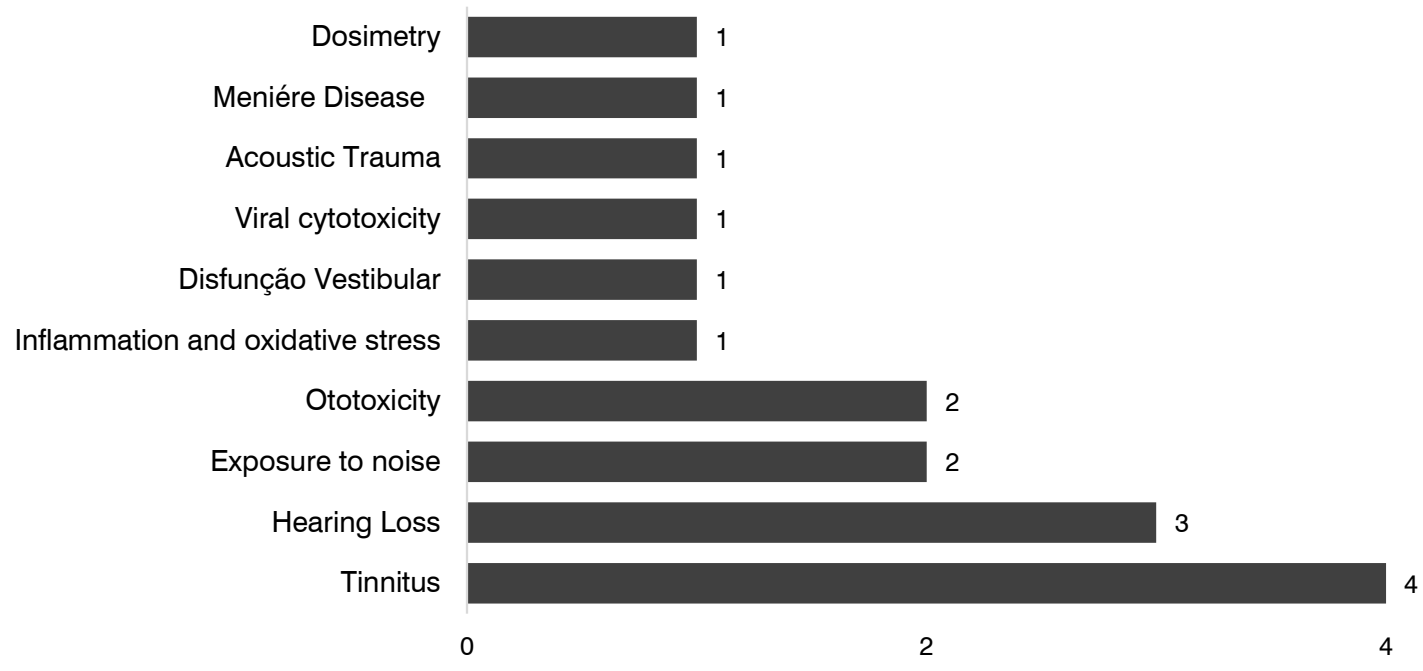

Figure 3: Demand for Laser Use.

location and structure of the inner ear that hinder the delivery of the gene or other therapeutic agents and also the type of cell used is extremely sensitive to damage, and under experimental conditions it is possible to induce oxidative stress by means of bacterial toxin or an ototoxic drug, enabling the investigation of the otoprotective effect of these cells after LLL irradiation ${ }^{35,36}$. Regarding the characterization of the laser used, there was a prevalence of the infrared laser $(n=8,57.1 \%)$, follow by red laser $(n=5,35,7 \%)$ and then multimodal laser $(n=1,7,1 \%)$, as according to some studies, exposure to low power, non-invasive infrared light at wavelengths ranging from $700 \mathrm{~nm}$ to $1400 \mathrm{~nm}$ has been effective in attenuating oxidative stress and overcoming inflammation in various organ systems and has a high penetration capacity when compared to other wavelengths ${ }^{37}$. Concerning the variation of the wavelength used in the $\mathrm{LBI}$, it was observed that there was a variation between $635 \mathrm{~nm}$ and $830 \mathrm{~nm}$, with emphasis on the length of $650 \mathrm{~nm}(\mathrm{n}=4$, 28.6\%) and 830nm $(\mathrm{n}=3,21,4 \%)$ and $810 \mathrm{~nm}(\mathrm{n}=3$, $21.4 \%$ ) (Figure 4). Several laboratory experiments and clinical trials used the laser in the red and infrared range and showed that they cause beneficial effects, since their penetration occurs from $\mathrm{mm}$ to a few centimeters, in addition to being able to excite intracellular molecules without causing damage to adjacent tissues ${ }^{38}$ (Figure 4).
In the analysis of the sample composition of the studies, the most recurrent composition was research carried out with rats $(n=8,64.3 \%)$, followed by humans $(n=$ $5,35.7 \%$ ), showing that many directions of research is still ongoing, and research is expanding. Animal studies have found that laser stimulation can induce anatomical and physiological changes in the cochlea ${ }^{39}$. In humans, the effects of TLBI on hearing loss and tinnitus have controversial results ${ }^{40,41}$. The conclusions of the articles, 13 searched to assess effectiveness while one searched to assess dosimetry. Among the 13 articles that evaluated the effectiveness, $78.1 \%(n=11)$ presented the LBI as effective for the treatment of inner ear problems ${ }^{42-47}$ already $14.2 \%$ of the articles $(n=2)$ presented the use of LBI with ineffective results ${ }^{48}$. Regarding the article that searched to evaluate dosimetry, it concluded that the effectiveness of the treatment depends on the choice of the ideal dose of light according to the location of application, however, other articles. Thus, it is noteworthy that the number of studies showing the effectiveness of TLBI is greater than those that had the opposite result. Among the most frequent limitations in the articles, the issue of sample size $(n=3)$ stands out, followed by the lack of a well-established protocol $(n=2)$ and the unproven effect $(n=2)$. Regarding the suggestions for future research, articles $(n=8)$ recommend that more studies 


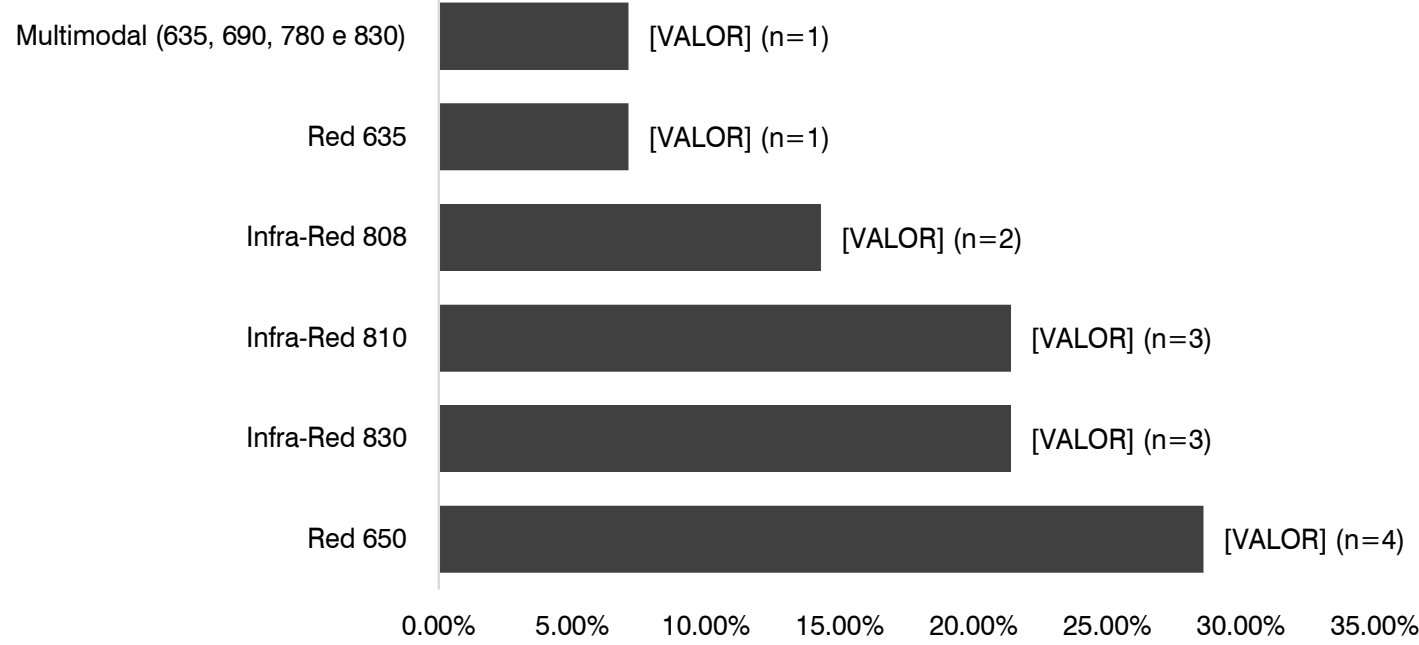

Figure 4: Wavelengths $(n=14)$.

should be carried out in the area in order to consolidate protocols and the results presented, there are still those that advise the elaboration of a universal application protocol with the parameters of use of the well-defined lasers $(n=3)$ and suggests that the sample should be enlarged in order for the results to be effective. All the articles analyzed contribute significantly to understanding the functioning of the LBI, as well as its application in the various areas of audiology. Despite the use of LBI in areas such as dentistry, medicine and physiotherapy, its use for audiology should be further studied, so that efficient and well-structured application protocols are created, thus contributing to the growth in the use of these therapies in the profession.

\section{CONCLUSION}

This study allowed quantifying and evaluating the most relevant articles on the topic of Audiology and Low Intensity Laser Therapy, enabling a better understanding of its use, applicability, and perspectives of using this tool for audiology. It was concluded that the interest in photobiomodulation therapy applied to audiology has grown in the last 4 years since the demand for noninvasive treatments has become a worldwide trend. The most frequently found objectives were regarding the evaluation of the effectiveness of this instrument in the face of problems related to hearing, such as tinnitus, vestibular dysfunctions and hearing loss, since the most common conclusions were about the real effectiveness of Low Level Laser (LLL) in audiological disorders. It was possible to conclude that the LLL is a tool of great value for professionals who take care of human hearing, since it helps in the recovery of patients with hearing problems in a non-invasive or medication way, enabling the improvement of their hearing functions. However, it was observed that there are no well-defined protocols on the applicability of LLL, which makes it difficult to replicate the studies found. Therefore, new studies on the effectiveness of LLL in audiological factors are suggested, as well as studies for the elaboration of well-defined protocols to spread knowledge among professionals in the field, facilitating the application of this therapy in patients with hearing problems.

\section{CONFLICT OF INTEREST}

The authors declare no potential conflict of interest.

\section{REFERENCES}

1. Correia R, Catanio A, Albuquerque I, Linhares M. Analysis of scientific production on hearing health in Brazil in four selected journals. SANARE Journal of Public Policies. 2014;13:99-109.

2. Aldergazly AA, Khlaif HR. Evaluation the Effect of Low Level Laser on Sensorineural Hearing Loss. Al-Nahrain J Eng Sci. 2018;1;21:384-8.

3. Hamill TA, Andrews JP. Audiology assistants in Private Practice, Semin Hear. 2016;1:348-58.

4. Taylor B. Interventional Audiology: Broadening the Scope of Practice to Meet the Changing Demands of the New Consumer, semin Hear. 2016;37:120-36.

5. Lee JH, Chang SY, Moy WJ, Oh C, Kim SH, Rhee CK, et al. Simultaneous bilateral laser therapy accelerates recovery after noise-induced hearing loss in a rat model. Peer $\mathrm{J}$. 2016;7:1-10.

6. Andrade FSSD, Clark RMO, Ferreira ML. Effects of lowlevel laser therapy on the healing of skin wounds. Brazilian College of Surgeons. 2014;1:129-33.

7. Fernanda GS, Juliana CS. Low-level Laser Therapy: A Review of Its Applications in the Management of Discovery Service for the University of Sao Paulo USP. Altern ther Health Med. 2016;22:24-31.

8. Cotler BH. The use of low level Laser Therapy (LLLT) for musculoskeletal pain. MOJ Orthop Rheumatol. 2015;9:2031.

9. Huang YY, Carroll J, Hamblin MR. Biphasic dose response in low level light therapy-an update. Former Nonlinearity Biol. 2011;9:602-18.

10. Hamblin MR. Mechanisms and applications of the antiinflammatory effects of photobiomodulation. AIMS Biophysics. American Institute of Mathematical Sciences. 2017;6:337-61. 
11. Consolim CF, Silva BPA, Sanches IC, Canal M, Consolim $\mathrm{CF}$, Conti FF, et al. Hemodynamic effect of laser therapy in spontaneously hypertensive rats. Arq Bras Cardiol. 2014;2:161-4.

12. Gomes CF, Schapochnik AO. Therapeutic use of the Low Intensity LASER (LBI) in some pathologies and its relationship with the performance in Speech Therapy. Community Disorders. 2017;29:570-80.

13. Rhee C, Song K, Chang SY, Jung JY, Lim SK, Chung PS, et al. defining a therapeutic dosage window for transmeatalLLLT applied to the rats with NIHL to Ameliorate NIHL. In: Photonic Therapeutics and Diagnostics. 2015;1:930-6.

14. Tamura A, Matsunobu T, Mizutari K, Niwa K, Kurioka T, Kawauchi S, et al. Low level laser therapy for prevention of noise-induced hearing loss in rats. Neurosci lett. 2015;9:595-8.

15. Bartos A, Grondin Y, Bortoni ME, Ghelfi E, Sepulveda $\mathrm{R}$, Carroll J, et al. Pre-conditioning with near infrared photobiomodulation reduces inflammatory cytokines and markers of oxidative stress in cochlear hair cells. J Biophotonics. 2016;1:1125-35.

16. Lee MY, Hyun JH, Suh MW, Ahn JC, Chung PS, Jung JY. Treatment of peripheral vestibular dysfunction using photobiomodulation. J Biomed Opt. 2017;29;22-46.

17. Nakashima T, Hiromi U, Hayato M, Suzuki T Tominaga $M$, Atsushi I et al. Transmeatal Low-power Laser Irradiation for Tinnitus : Otology \& Neurotology. 2002;23:296-300.

18. Teggi R, Bellini C, Piccioni LO, Palonta F, Bussi M. Transmeatal low-level laser therapy for chronic tinnitus with cochlear dysfunction. Audiol Neurotol. 2009; 14:115-20.

19. Ngao CF, Tan TS, Narayanan P, Raman R. The effectiveness of transmeatal low-power laser stimulation in treating tinnitus. Eur Arch Oto-Rhino-Laryngology. 2014;271:975-80.

20. Shiomi Y, Takahashi H, Honjo I, Kojima H, Naito Y, Fujiki N. Efficacy of transmeatal low power laser irradiation on tinnitus: A preliminary report. Auris Nasus Larynx. 1997;24:39-42.

21. Gungor A, Dogru S, Cincik H, Erkul E, Poyrazoglu E. Effectiveness of transmeatal low power laser irradiation for chronic tinnitus. J Laryngol Otol. 2008;122:447-51.

22. Okhovat A, Berjis $N$, Okhovat $H$, Malekpour A, Abtahi $H$. Low-level laser for treatment of tinnitus: a self-controlled clinical trial. J Res Med Sci. 2011;16:33-36.

23. Mollasadeghi A, Mirmohammadi SJ, Mehrparvar AH, Davari $\mathrm{MH}$, Shokouh $\mathrm{P}$, Mostaghaci M, et al. Efficacy of low-level laser therapy in the management of tinnitus due to noiseinduced hearing loss: A double-blind randomized clinical trial. Sci World J. 2013;20:13-26.

24. Choi JE, Lee MY, Chung PS, Jung JY. A preliminary study on the efficacy and safety of low level light therapy in the management of cochlear tinnitus: A single blind randomized clinical trial. Int Tinnitus J. 2019;1:52-7.

25. Gomes CF, Schapochnik AO. therapeutic use of the Low Intensity LASER (LBI) in some pathologies and its relationship with the performance in Speech Therapy. Community Disorders. 2017;29:570-6.

26. Rezende SK, Miceli Kerbauy MT. Quanti-qualitative approach: overcoming the quantitative-qualitative dicotoamy in research in education. Educ E Filos. 2017;31:21-44.
27. Quevedo-Silva B, Almeida Santos B, Brandão M. Brazilian Magazine of Marketing. 2016;11:246-62.

28. Witwyt LP. Speech, Language and Hearing Sciences and public health: bibliometric analysis Speech therapy and public health: bibliometric analysis Speech therapy and public health: bibliometric analysis. 2017;29:227-36.

29. Goodman SS, Bentler RA, Dittberner A, Mertes I. The Effect of Low-Level Laser Therapy on Hearing. ISRN Otolaryngol. 2013;1:1-9.

30. Peng Z, Chen XQ, Gong SS, Chen CF. Low-level laser therapy for tinnitus. Cochrane Database Syst Rev. 2014;20:14-28.

31. Gunther H. Qualitative Research Versus Quantitative Research: Is This the Question ?. Psychology: Theory and Research. 2006;6:201-10.

32. Aldergazly A, Khlaif $H$. Evaluation the Effect of Low Level Laser on Sensori-neural Hearing Loss. Al-Nahrain J Eng Sci. 2018;1:21-84.

33. Lee JH, Kim S, Jung JY, Lee MY. Applications of photobiomodulation in hearing research: from bench to clinic. Biomed Eng Lett. 2019;9:351-8.

34. Tauber S, Baumgartner R, Schorn K, Beyer W. Light dosimetric quantitative analysis of the human petrous bone: Experimental study for laser irradiation of the cochlea. Lasers Surg Med. 2001;28:18-26.

35. Bartos A, Grondin Y, Bortoni ME, Ghelfi E, Sepulveda $\mathrm{R}$, Carroll J, et al. Pre-conditioning with near infrared photobiomodulation reduces inflammatory cytokines and markers of oxidative stress in cochlear hair cells. J Biophotonics. 2016;1:1125-35.

36. Chang SY, Park YH, Carpena NT, Pham TT, Chung PS, Jung JY, et al. Photobiomodulation promotes adenoviral gene transduction in auditory cells. Lasers Med Sci. 2019;34:36775.

37. Lee MY, Bae SH, Chang SY, Lee JH, Kim SH, Ahn JC, et al. Photobiomodulation by laser therapy rescued auditory neuropathy induced by Cuabin. Neurosci Lett. 2016;28:16573.

38. Elsanadiky $H$, Nafie $Y$. Evaluation of transient evoked otoacoustic emissions using low-level laser stimulation in individuals with normal hearing with tinnitus. Tanta Med J. 2017;1:45-66.

39. Lee JH, Lee MY, Chung PS, Jung JY. Photobiomodulation using low-level $808 \mathrm{~nm}$ diode laser rescues cochlear synaptopathy after acoustic overexposure in rat. $J$ Biophotonics. 2019;12:1-10.

40. Mollasadeghi A, Mirmohammadi SJ, Mehrparvar AH, Davari $\mathrm{MH}$, Shokouh $\mathrm{P}$, Mostaghaci M, et al. Efficacy of low-level laser therapy in the management of tinnitus due to noiseinduced hearing loss: A double-blind randomized clinical trial. Sci World J. 2013;2:13-56.

41. Dehkordi MA, Einolghozati S, Ghasemi SM, Abolbashari S, Meshkat M, Behzad $\mathrm{H}$. Effect of low-level laser therapy in the treatment of cochlear tinnitus: A double-blind, placebocontrolled study. 2015;94:32-6.

42. Tamura A, Matsunobu T, Mizutari K, Niwa K, Kurioka T, Kawauchi S, et al. Low-level laser therapy for prevention of noise-induced hearing loss in rats. Neurosci Lett. 2015;9:81-6. 
43. Lee MY, Hyun JH, Suh MW, Ahn JC, Chung PS, Jung JY. Treatment of peripheral vestibular dysfunction using photobiomodulation. J Biomed Opt. 2017; 29:22-8.

44. Rhee CK, He P, Jung JY, Ahn JC, Chung PS, Suh MW. Effect of low-level laser therapy on cochlear hair cell recovery after gentamicin-induced ototoxicity. Lasers Med Sci. 2012;27:987-92.

45. Rhee CK, Bahk CW, Kim SH, Ahn JC, Jung JY, Chung PS, et al. Effect of low-level laser treatment on cochlea haircell recovery after acute acoustic trauma. J Biomed Opt. 2012;17:68-102.
46. Teggi R, Bellini C, Fabiano B, Bussi M. Efficacy of lowlevel laser therapy in Ménière's disease: A pilot study of 10 patients. Photomed Laser Surg. 2008;26:349-53.

47. Tauber S, Beyer W, Schorn K, Baumgartner R. Transmetal Cochlear Laser (TCL) treatment of cochlear dysfunction: A feasibility study for chronic tinnitus. Lasers Med Sci. 2003;18:154-61.

48. Teggi R, Bellini C, Piccioni LO, Palonta F, Bussi M. Transmetal low-level laser therapy for chronic tinnitus with cochlear dysfunction. Audiol Neurotol. 2009; 14:115-20. 